\title{
An anatomically realistic lung model for Monte Carlo-based dose calculations
}

\author{
Liang Liang ${ }^{\text {a) }}$ and Edward W. Larsen \\ Department of Nuclear Engineering and Radiological Sciences, University of Michigan, Ann Arbor, \\ Michigan 48109-2104 \\ Indrin J. Chetty \\ Department of Radiation Oncology, University of Nebraska Medical Center, Omaha, Nebraska 68198-7521
}

(Received 15 June 2006; revised 6 January 2007; accepted for publication 8 January 2007; published 22 February 2007)

Treatment planning for disease sites with large variations of electron density in neighboring tissues requires an accurate description of the geometry. This self-evident statement is especially true for the lung, a highly complex organ having structures with a wide range of sizes that range from about $10^{-4}$ to $1 \mathrm{~cm}$. In treatment planning, the lung is commonly modeled by a voxelized geometry obtained using computed tomography (CT) data at various resolutions. The simplest such model, which is often used for QA and validation work, is the atomic mix or mean density model, in which the entire lung is homogenized and given a mean (volume-averaged) density. The purpose of this paper is (i) to describe a new heterogeneous random lung model, which is based on morphological data of the human lung, and (ii) use this model to assess the differences in dose calculations between an actual lung (as represented by our model) and a mean density (homogenized) lung. Eventually, we plan to use the random lung model to assess the accuracy of CT-based treatment plans of the lung. For this paper, we have used Monte Carlo methods to make accurate comparisons between dose calculations for the random lung model and the mean density model. For four realizations of the random lung model, we used a single photon beam, with two different energies $(6$ and $18 \mathrm{MV})$ and four field sizes $\left(1 \times 1,5 \times 5,10 \times 10\right.$, and $\left.20 \times 20 \mathrm{~cm}^{2}\right)$. We found a maximum difference of $34 \%$ of $D_{\max }$ with the $1 \times 1,18 \mathrm{MV}$ beam along the central axis (CAX). A "shadow" region distal to the lung, with dose reduction up to $7 \%$ of $D_{\max }$, exists for the same realization. The dose perturbations decrease for larger field sizes, but the magnitude of the differences in the shadow region is nearly independent of the field size. We also observe that, compared to the mean density model, the random structures inside the heterogeneous lung can alter the shape of the isodose lines, leading to a broadening or shrinking of the penumbra region. For small field sizes, the mean lung doses significantly depend on the structures' relative locations to the beam. In addition to these comparisons between the random lung and mean density models, we also provide a preliminary comparison between dose calculations for the random lung model and a voxelized version of this model at $0.4 \times 0.4 \times 0.4 \mathrm{~cm}^{3}$ resolution. Overall, this study is relevant to treatment planning for lung tumors, especially in situations where small field sizes are used. Our results show that for such situations, the mean density model of the lung is inadequate, and a more accurate CT model of the lung is required. Future work with our model will involve patient motion, setup errors, and recommendations for the resolution of CT models. (C) 2007 American Association of Physicists in Medicine. [DOI: 10.1118/1.2437284]

Key words: lung model, dosimetry, Monte Carlo, inhomogeneity correction

\section{INTRODUCTION}

Tissue inhomogeneity corrections are necessary for treatment planning in sites such as the lung. ${ }^{1-7}$ Previous work ${ }^{8-16}$ modeled the lung as a homogenized mixture of tissue and air, at a lower density than the surrounding tissue, in order to gain understanding of certain inhomogeneity effects between the lung and surrounding tissue. This homogeneous model is also called the atomic mix ${ }^{17}$ or mean density model. However, the lung is a highly complex organ, consisting of "chunks" of tissue and air ranging in diameter from about $10^{-4}$ to $1.0 \mathrm{~cm}$, with millions of air-tissue interfaces, ${ }^{18,19}$ and it is not obvious that the mean density model should be ac- ceptable for treatment planning. In fact, modern treatment planning uses computed tomography (CT)-based patient geometry, in which the voxels are relatively small local homogenized volumes with varying densities and compositions. However, the resolution at which one can adequately represent the lung remains an open question. In this paper, we (i) propose a realistic heterogeneous model of the lung, and (ii) present some preliminary Monte Carlo (MC) calculations that compare this model to the mean density model and a single voxelized version of the original random lung. We find that in some important situations, dose is not well predicted by the mean density or CT models. In future work, we plan to use the random lung model to systematically assess the 
adequacy of current methods for treatment planning in the lung.

The human lung is a spongy, heterogeneous organ consisting of two materials of great density variation: air and tissue. The relative positions and local composition of these two materials are patient specific and time dependent, showing a feature of unpredictable "randomness." Traditionally, a heterogeneous particle transport region may be accurately treated by the mean density model if a typical chunk size in the region is smaller than the mean free path (MFP) of the particle. ${ }^{17,20}$ For megavoltage photons with a MFP of tens of $\mathrm{cm}$, the lung's structure is sufficiently fine to be treated by the mean density model. However, dose deposition is a twostep process: (i) charged particles are generated by interactions between incident photons and irradiated matter; and (ii) these charged particles deposit their kinetic energy along their flight path. The charged particles set in motion by megavoltage photons have a range on the order of centimeters and a MFP on the order of microns. Under charged particle equilibrium (CPE), the charged particles can be thought to deposit all their energy locally; only the MFPs of photons matter, and the mean density approximation is valid. However, for situations where CPE does not exist, such as within a small beam, or near a beam's edge or a material interface, the fact that the size of even the lung's finest structures is comparable to the charged particles' MFP becomes an important consideration. In these conditions, the mean density approximation is no longer guaranteed to be valid, and the random lung structure could lead to perturbations in the dose distribution. The actual dose would then deviate from that obtained from the mean density lung model.

In this study we develop a simplified but geometrically sound heterogeneous random lung model, based on morphological data of the human lung. We use the Monte Carlo method to perform dose calculations for the "random" and "mean density" lung models, because Monte Carlo is known to yield highly accurate dose distributions for generally heterogeneous systems. We also use the Monte Carlo method to compare, in a preliminary simulation, the random lung model and one of its "voxelized" versions. We find that the mean density and voxelized approximations to the random lung model can be inadequate, particularly for small field sizes.

The remainder of this paper is organized as follows: in Sec. II we describe the lung model, and in Sec. III we describe our MC simulations. Section IV presents our results and discussion, and Sec. V is a conclusion and summary.

\section{THE 2 1/2D LUNG MODEL}

\section{A. Morphology of the lung}

The human lung is composed basically of two materials of dramatically different densities (tissue and air), with random local composition and a wide range of chunk sizes, ranging from the order of $1.0 \mathrm{~cm}$ (principle bronchi, main pulmonary arteries, and veins) down to $10^{-4} \mathrm{~cm}$ (alveolus structures). In general, human lungs consist of tube-like conducting airways, circulation pathways, and very finely struc- tured parenchymal tissue. The conducting airways bifurcate repeatedly and form the nearly dichotomous bronchial tree from the carina. Similarly, the vessels form the bifurcating pulmonary arterial and venous trees, respectively. The morphology of these trees has been well studied over the last several decades by measuring prepared casts of the airways/ vessels, and by visualizing and analyzing the trees on in vivo CT scans. ${ }^{21-27}$

The Horsfield order, ${ }^{22}$ a numbering system starting from the lung's periphery and working toward the stem, is used to group different generations (labeling the branches in the reverse direction of "order") of branches of the bifurcating bronchial and arterial/venous trees (Table I). Each order has its number, typical size, and other morphological characteristics, such as the total number of the branches of this order in the whole lung.

The bronchi are accompanied by the pulmonary arteries, while the veins are separate from these. The " $T / D$ ratio" is the wall thickness $(T)$ divided by the total diameter of the bronchus $(D)$, and the "bronchoarterial ratio" is the diameter of the bronchial lumen $(D+2 T)$ divided by its accompanying pulmonary artery. One study ${ }^{28}$ showed that the $T / D$ ratio has no statistically significant difference between segments, lobes and lungs. Furthermore, no significant correlation was shown between $T / D$ ratio and age, while the bronchoarterial ratio showed a significant correlation with age. The purely conducting airways of the bronchial tree start from the right/ left principal bronchus, with a typical luminal diameter of $1.2 \mathrm{~cm}$, and end at terminal bronchioles, with an internal diameter in the range of $0.03-0.1 \mathrm{~cm} .{ }^{18}$ Distal to each terminal bronchiole is the acinus: the complex of alveolated airways and the largest parenchymal unit, which contains three generations (on average) of respiratory bronchioles and numerous alveoli where gas exchange mainly occurs. The acinus has an average volume of $187 \mathrm{~mm}^{3}$ and numbers $26000-32000$ in both lungs, assuming a total lung capacity of $5-61 .^{25}$ The internal airway diameter inside an acinus falls from 0.05 to $0.027 \mathrm{~cm}$. ${ }^{25}$ The end structure containing air is the thin-walled pouch-like alveolus, with a mean diameter of about $0.025 \mathrm{~cm} .^{18}$ The total number of alveoli in each adult lung ranges from $200 \times 10^{6}$ to $600 \times 10^{6}$, depending on body size. ${ }^{18}$

\section{B. Lung model}

\section{Threshold size}

Due to the extreme geometrical complexity of the lung, it is not practical to build a real lung model down to the smallest order of the hierarchical structures and simulate this model in Monte Carlo calculations. Fortunately, a theoretical part of our work ${ }^{29}$ indicates that we can employ a simplified model that (i) retains structures of sizes larger than a threshold size, and (ii) homogenizes all structures finer than the threshold size into a homogeneous mean density background. The threshold size should be (i) sufficiently small that in regions with no structures larger than this threshold size, the dose distribution is nearly the same as the dose 
TABLE I. Morphometric data (Refs. 22 and 24) of bronchial, arterial and venous trees and the number $n$ (number of structures in the lung) and $m$ (number of structures in the model) used in developing the heterogeneous random 2 1/2D lung model.

\begin{tabular}{|c|c|c|c|c|c|c|}
\hline $\begin{array}{c}\text { Horsfield } \\
\text { order }\end{array}$ & $\begin{array}{l}\text { Length } \\
(\mathrm{mm})\end{array}$ & $\begin{array}{l}\text { Lumen } \\
\text { diameter } \\
(\mathrm{mm})\end{array}$ & $\begin{array}{l}\text { Bronchus } \\
\text { diameter } \\
(\mathrm{mm})\end{array}$ & $\begin{array}{c}\text { Artery/vein } \\
\text { diameter } \\
(\mathrm{mm})\end{array}$ & $n$ & $m$ \\
\hline 28 & 100 & 16 & 26.67 & 22.86 & 0 & 0 \\
\hline 27 & 40 & 12 & 20.00 & 17.14 & 0 & 0 \\
\hline 26 & 26 & 10.3 & 17.17 & 14.71 & 2 & 1.86 \\
\hline 25 & 18 & 8.9 & 14.83 & 12.71 & 2 & 1.29 \\
\hline 24 & 14 & 7.7 & 12.83 & 11.00 & 2 & 1.00 \\
\hline 23 & 11 & 6.6 & 11.00 & 9.43 & 3 & 1.18 \\
\hline 22 & 10 & 5.7 & 9.50 & 8.14 & 6 & 2.14 \\
\hline 21 & 10 & 4.9 & 8.17 & 7.00 & 8 & 2.86 \\
\hline 20 & 10 & 4.2 & 7.00 & 6.00 & 12 & 4.29 \\
\hline 19 & 10 & 3.5 & 5.83 & 5.00 & 14 & 5.00 \\
\hline 18 & 9.6 & 3.3 & 5.50 & 4.71 & 20 & 6.86 \\
\hline 17 & 9.1 & 3.1 & 5.17 & 4.43 & 30 & 9.75 \\
\hline 16 & 8.6 & 2.9 & 4.83 & 4.14 & 37 & 11.36 \\
\hline 15 & 8.2 & 2.8 & 4.67 & 4.00 & 46 & 13.47 \\
\hline 14 & 7.8 & 2.6 & 4.33 & 3.71 & 64 & 17.83 \\
\hline 13 & 7.4 & 2.4 & 4.00 & 3.43 & 85 & 22.46 \\
\hline 12 & 7 & 2.3 & 3.83 & 3.29 & 114 & 28.50 \\
\hline 11 & 6.7 & 2.2 & 3.67 & 3.14 & 158 & 37.81 \\
\hline 10 & 6.3 & 2 & 3.33 & 2.86 & 221 & 49.72 \\
\hline 9 & 5.7 & 1.78 & 2.97 & 2.54 & 341 & 69.42 \\
\hline 8 & 5 & 1.51 & 2.52 & 2.16 & 499 & 89.11 \\
\hline 7 & 4.4 & 1.29 & 2.15 & 1.84 & 760 & 119.43 \\
\hline 6 & 3.9 & 1.1 & 1.83 & 1.57 & 1104 & 153.77 \\
\hline 5 & 3.5 & 0.93 & 1.55 & 1.33 & 1675 & 209.38 \\
\hline 4 & 3.1 & 0.79 & 1.32 & 1.13 & 2843 & 314.76 \\
\hline 3 & 1.1 & 0.64 & 1.07 & 0.91 & 5651 & 222.00 \\
\hline 2 & 1.3 & 0.56 & 0.93 & 0.80 & 11300 & 524.64 \\
\hline 1 & 1.1 & 0.51 & 0.85 & 0.73 & 25000 & 982.14 \\
\hline
\end{tabular}

distribution in the homogenized model, and (ii) as large as possible, to minimize the complexity of the geometry and the cost of the Monte Carlo simulations.

The classic atomic mix (mean density) approximation states that in a geometrically random system in which the chunk sizes are small compared to a mean free path, one can replace the geometrically random system by the homogenized mean-density system, and the resulting dose will accurately match the dose for the original system. ${ }^{17,20}$ To apply this classic approximation, the threshold size should be on the order of a mean free path for the radiation delivering the dose (the electrons). Unfortunately, the electron mean free path is so small that this would require almost the entire geometrical structure of the lung to be explicitly modeled; doing this would be prohibitively costly.

However, our theoretical work has shown that the classic atomic mix approximation can be greatly extended for radiation with highly forward-peaked scattering (in particular, electrons). ${ }^{29}$ Specifically, we have shown that the atomic mix approximation is valid for a random system in which the chunk sizes are small compared to a transport mean free path $\lambda_{\text {tr }}$, which is defined as ${ }^{30,31}$

$$
\lambda_{\mathrm{tr}}=\frac{\lambda}{1-\langle\mu\rangle},
$$

where $\lambda$ is the mean free path and $\langle\mu\rangle$ is the mean scattering cosine. This result implies that it is acceptable to choose a threshold size on the order of an electron transport mean free path, which because $\langle\mu\rangle \approx 1$, can be orders of magnitude greater than an electron mean free path. This extension of the classic atomic mix approximation makes it feasible to construct a practical model of the lung for accurately assessing dose deposited by photon and electron beams.

For the lowest electron energies treated, the electron transport mean free path in water is about $0.05 \mathrm{~cm}$, which is about the size of the terminal bronchiole. In our model, all structures smaller than $0.05 \mathrm{~cm}$ are homogenized to a uniform "background" with an adjusted mean density. This part of the lung is called the parenchyma; it contains about $90 \%$ of the total lung volume (with structures typically $0.01 \mathrm{~cm}$ in diameter) and about $70 \%$ of the lung mass. All the larger structures in our random lung model are explicitly modeled.

We have tested this concept in detailed Monte Carlo simulations and have found that the theoretical prediction-that 


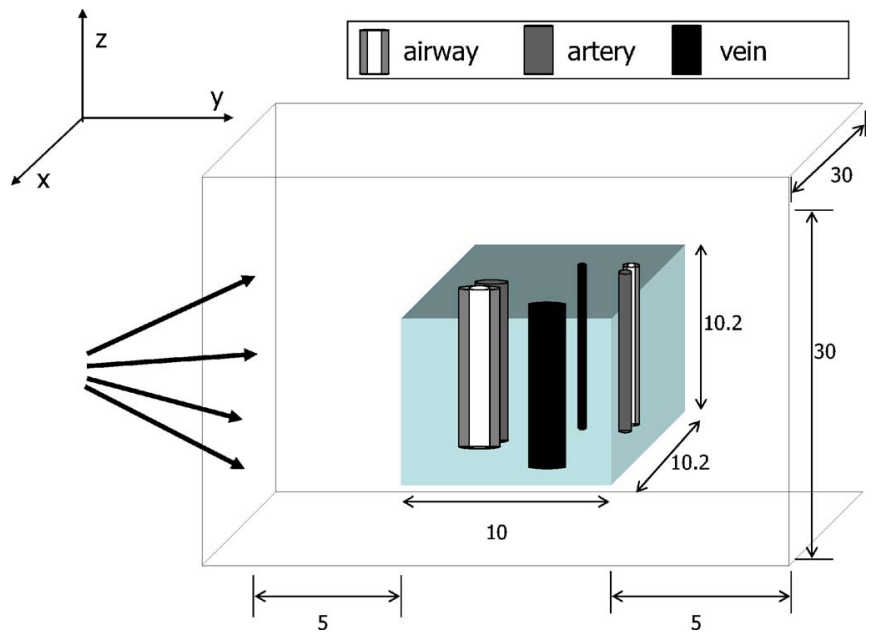

FIG. 1. Three-dimensional view of the simulated phantom geometry and corresponding dimensions $(\mathrm{cm})$ with the $21 / 2 \mathrm{D}$ lung model embedded inside the surrounding water. The coordinate system is shown in the upper left corner; the photon beams are incident in the positive $y$ direction and perpendicular to the $x-z$ plane; the upper legend illustrates modeled airways (concentric cylinders), arteries (single cylinders attached to the airways in the lung) and veins (independent single cylinders in the lung) inside the lung.

electron transport in a geometrically random system with chunk sizes smaller than a transport mean free path is accurately modeled by atomic mix - is highly accurate. In particular, Monte Carlo simulations of photon and electron transport in a random lung model with a threshold size of $0.05 \mathrm{~cm}$ are also highly accurate. ${ }^{32}$

\section{2. "Random" 2 1/2D geometry}

In our model, we did not duplicate the bronchial/arterial/ venous trees down to the terminal bronchioles, arterioles and venules in their three-dimensional (3D) form. Instead, to make our model as simple as possible for MC simulations, yet geometrically sound, a "random" 2 1/2D model was proposed, which is essentially a 2D geometry extending a finite distance in one dimension ( $z$ direction in the simulation coordinates) and cut to fit into the simulated lung region (see Fig. 1). Due to the fact that the airway element has the approximate shape of a hollow cylinder, ${ }^{21}$ in this model, the "airways" and the "arteries/veins" are modeled as randomly positioned cylinders with axes parallel to the $z$ direction (Fig. 1). Each airway consists of two concentric cylinders with the outer cylinder being the wall. The radius of the airway lumen for each order was taken directly from the available morphological data, and the wall thickness was calculated from the $T / D$ ratio. An artery/vein is taken to be a solid cylinder. Based on the information stated above, an airway is always attached to the artery at a randomly chosen position, while the vein of the same order has a positive distance to the airway/artery bundle. Because of the lack of data on this distance, we assumed that the veins of the last seven simulated orders (including all lobar and broncho-pulmonary segment bronchi) should stay in close proximity to the airways/ arteries of the same order before entering the broncho- pulmonary segments. In our model, the distance between the center of the vein and the center of the smallest circle containing the airway/artery bundle (the circumcircle) is set to double the radius of this circumcircle. The position is randomly selected around the bundle. The remaining smaller orders of veins have no such restriction on position and are uniformly distributed within the model. Arteries and veins are assumed to have the same number of orders as the airways, and to have equal radii, which were computed from the bronchoarterial ratio for the same order. Values computed under this assumption are consistent with the results of a morphological study. ${ }^{26}$ Representative top views in the $x-y$ plane are given in Fig. 2.

\section{Relevant geometrical and physical parameters}

An important feature of our model is that it conserves the volume ratio of each order of structure, in such a way that the mass for the entire lung (not local regions such as the four particular realizations which will be discussed in Sec. III.) is the same for both the homogeneous (mean density) and the heterogeneous (random 2 1/2D) models. To achieve this goal, two quantities need to be computed correctly. One is the adjusted numbers of structures for each order in the 2 $1 / 2 \mathrm{D}$ geometry. The other is the adjusted mass density for the homogenized part (parenchyma) of the $21 / 2 \mathrm{D}$ model.

We used the following equation to map the number $n$ of the structures in a specific order from a real lung to the number $m$ of the same order in our $21 / 2 \mathrm{D}$ model, based on the morphometric data in Table I:

$$
\frac{m_{\text {structure }}^{\text {model }} \cdot a_{\text {structure }}}{S_{\text {model }}}=\frac{n_{\text {structure }}^{\text {lung }} \cdot v_{\text {structure }}}{V_{\text {lung }}} .
$$

Here we have defined:

$m_{\text {structure }}^{\text {model }} \quad$ number of structures of a specific order in the model,


real lung,

$a_{\text {structure }} \quad$ cross section of the structure,

$v_{\text {structure }} \quad$ volume of the structure,

$S_{\text {model }} \quad$ area chosen to be able to generate all orders of structures,

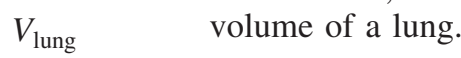

In Eq. (1), $S_{\text {model }}$ determined in such a way that the smallest calculated $m$ is greater or equal to 1, i.e., this order of structures must appear at least once in the model geometry. $V_{\text {lung }}$ is set to a typical $3000 \mathrm{ml}$ in this study. The calculated $m$ 's are presented in Table I in which most of the $m$ 's are not whole numbers. Since the structures in the geometry cannot be fractional, a new $m$ was recalculated by generating a random number $\xi$. If $\xi<m-[m], m=m+1$; otherwise, $m=m$. We note that the last two orders of largest structures were not included in the model because these two are the trachea and the principal bronchus, which are not part of the lung. The minimum value of $S_{\text {model }}$ which allows at least one structure from each order is $2143 \mathrm{~cm}^{2}\left(46.3 \times 46.3 \mathrm{~cm}^{2}\right)$. We construct 

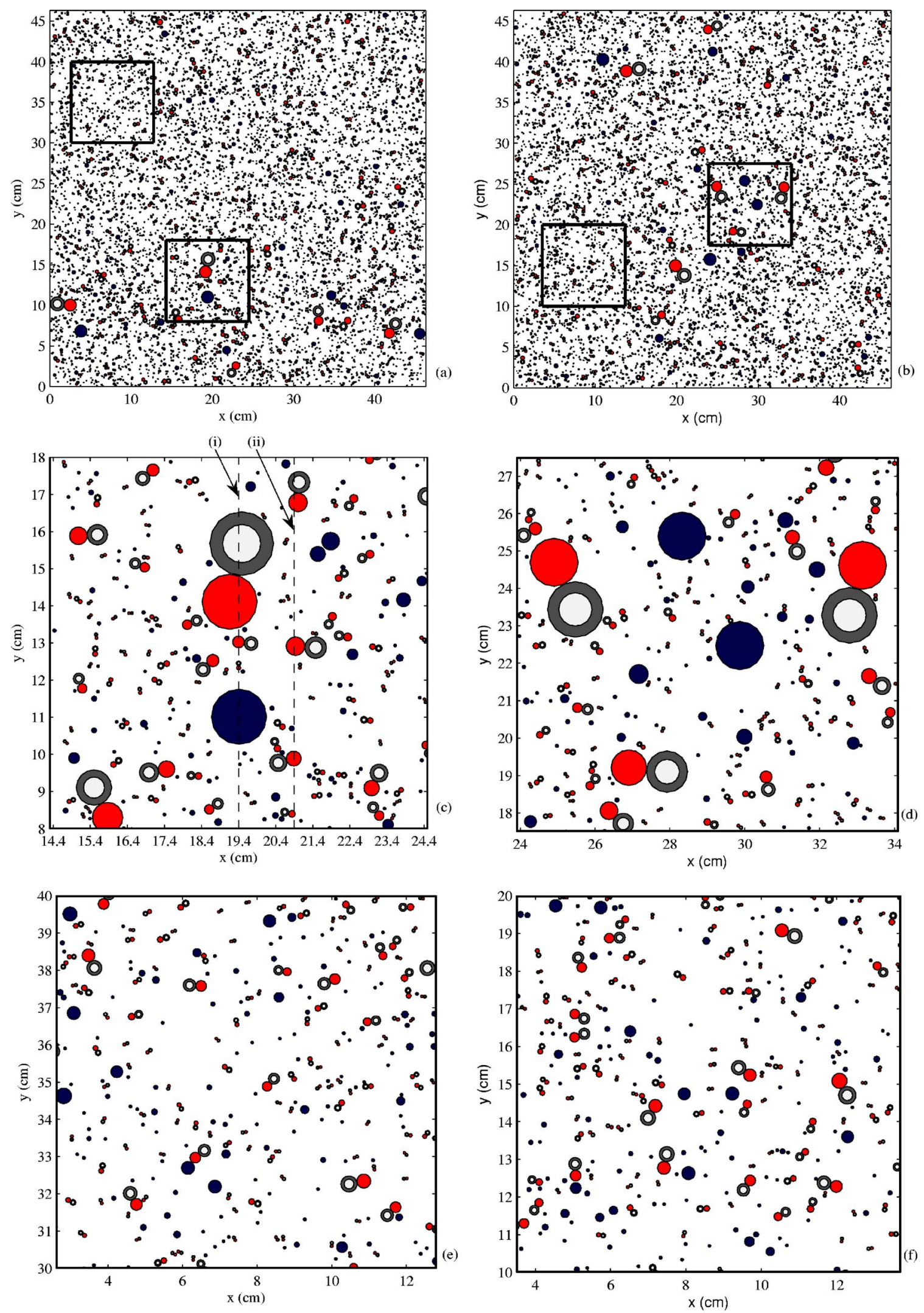

FIG. 2. Top view of the random heterogeneous lung model in which the concentric circles represent bronchi, the single circles attached to the bronchi are arteries, and the independent single circles are veins: (a-b) two realizations of the modeled "whole" lung, with four selected $10.2 \times 10 \mathrm{~cm}^{2}$ regions to fit in the lung block in the simulated phantom (see also Fig. 1), respectively; (c) realization $L_{1}$, indicated in (a) by the lower right framed region, with one large bronchus, artery and vein close to the CAX. [Lines (i) and (ii) are used in Fig. 3]; (d) realization $L_{2}$, indicated in (b) by the upper right framed region, with two large bronchi, arteries, and veins off the CAX; (e) realization $S_{1}$, indicated in (a) by the upper left framed region, with no large structures; (f) realization $S_{2}$, indicated in (b) by the lower left framed region, with no large structures. 
"whole lung realizations" using this value of $S_{\text {model }}$ but then select $10.2 \times 10 \mathrm{~cm}^{2}$ subregions to represent an actual lung. This is illustrated in Fig. 2.
The density of the background homogeneous parenchyma is computed using Eq. (2)

$$
\rho_{\text {parenchyma }}=\frac{\rho_{\text {lung }} V_{\text {lung }}-\sum_{\text {order }} n_{\text {order }}\left[\rho_{\text {air }} v_{\text {order }}^{\text {lumen }}+\rho_{\text {water }}\left(v_{\text {order }}^{\text {wall }}+v_{\text {order }}^{\text {artery }}+v_{\text {order }}^{\text {vein }}\right)\right]}{V_{\text {lung }}-\sum_{\text {order }} n_{\text {order }}\left[v_{\text {order }}^{\text {lumen }}+v_{\text {order }}^{\text {wall }}+v_{\text {order }}^{\text {artery }}+v_{\text {order }}^{\text {vein }}\right]} .
$$

where $\rho=$ density, and other parameters are defined in Eq. (1).

The mean density of the lung can vary significantly among different people. $^{33}$ We set $\rho_{\text {lung }}=0.26 \mathrm{~g} / \mathrm{cm}^{3}$; $\rho_{\text {parenchyma }}$ as then $0.201 \mathrm{~g} / \mathrm{cm}^{3}$ accordingly. The $T / D$ ratio 0.2 , and the bronchoarterial ratio 0.695 conforms to measured data. ${ }^{28}$ We used four different densities of water as four materials appearing in the geometry: (i) water of density $1.0 \mathrm{~g} / \mathrm{cm}^{3}$ as the matter of the airway wall, artery and vein, as well as that of the phantom outside the lung; (ii) water of density $0.26 \mathrm{~g} / \mathrm{cm}^{3}$ as the homogenized (mean density) lung; (iii) water of density $0.201 \mathrm{~g} / \mathrm{cm}^{3}$ as the lung parenchyma (the background); and (iv) water of density $0.00120479 \mathrm{~g} / \mathrm{cm}^{3}$ as the air inside the airways. We used only water composition for all different tissues in order to eliminate any factors that might affect the dose calculation other than the random geometry itself. The line density change in the lung along the $y$ direction at two different width is depicted in Figs. 3(a) and 3(b). These figures show the major differences in local densities between the mean density and the heterogeneous lung models.

\section{Voxelization}

To mimic the CT scan, we also voxelize this detailed random lung by superimposing a rectilinear grid on it and calculating the mass/density accordingly for each voxel, and homogenizing the material with each spatial cell (voxel). In this way, we obtain a voxelized random lung phantom, in which the density within each voxel is uniform, but the density generally varies from one voxel to the next. The resulting voxelized random lung model is analogous to the lung models obtained from CT data. Figure 4 shows the voxelized version (top view in the $x-y$ plane) of Fig. 2(c) at a resolution of $0.4 \times 0.4 \times 0.4 \mathrm{~cm}^{3}$.

\section{MONTE CARLO SIMULATIONS}

The Monte Carlo code PENELOPE ${ }^{34}$ was employed for most of the calculations in this study. We simulated the open field $x$ ray from a point source, with two clinical photon beam spectra ( 6 and $18 \mathrm{MV}$, which were calculated by Sheikh-Bagheri and Rogers ${ }^{35}$ for the Varian Clinac) and four field sizes $\left(1 \times 1,5 \times 5,10 \times 10\right.$, and $\left.20 \times 20 \mathrm{~cm}^{2}\right)$ at an $\mathrm{SSD}=$ source-surface distance $=100 \mathrm{~cm}$. The photon fluence at the entrance surface of the phantom was uniform across the field. Cutoff energies of $100 \mathrm{keV}$ for electrons/positions and $20 \mathrm{keV}$ for photons were used throughout. The photon transport is performed with analog Monte Carlo. The electron transport is performed with the condensed history method, using step sizes sufficiently small that at least five
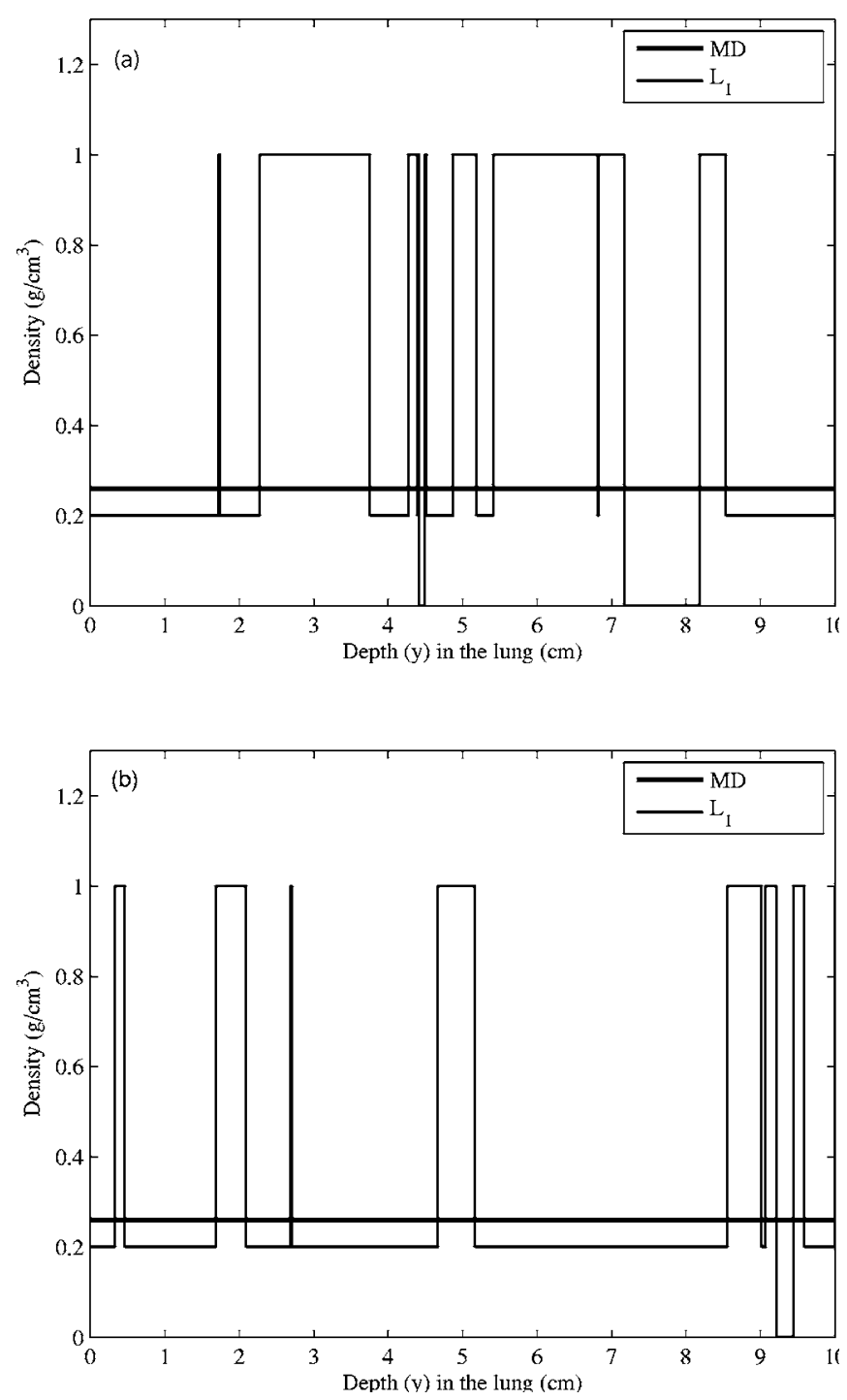

FIG. 3. Line density change in $y$ direction in the lung. The thick lines depict the uniform mean density (MD) case. The thin lines are for the heterogeneous realization $L_{1}$ in Fig. 2(c): (a) corresponds to dashed line (i) and (b) corresponds to dashed line (ii). 


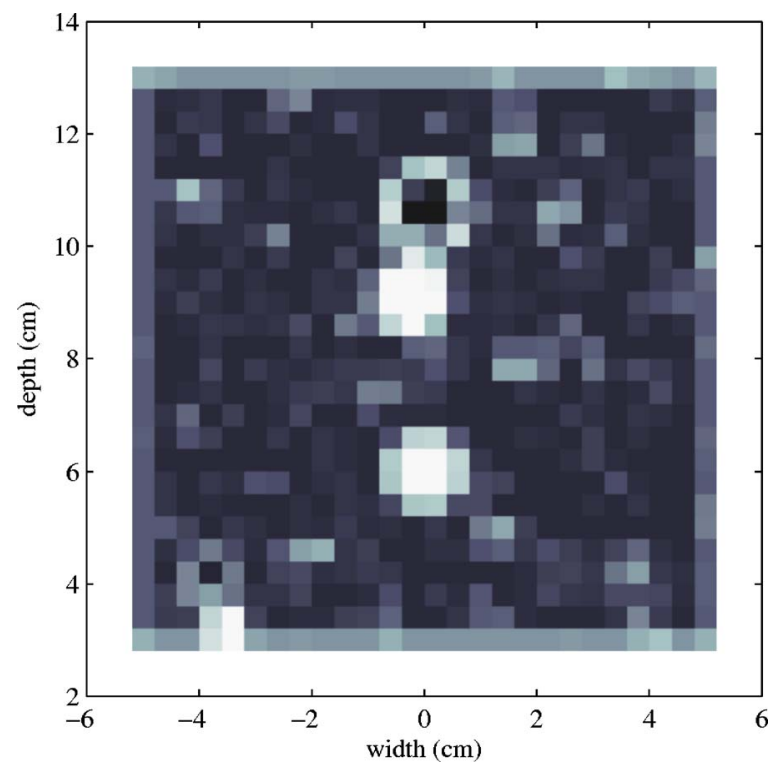

FIG. 4. Top view of a voxelized version of realization $L_{1}$ as shown in Fig. 2(c) to mimic the CT scan. The resolution is set to be $0.4 \times 0.4 \times 0.4 \mathrm{~cm}^{3}$.

steps are required to transport electrons through each chunk. Dose scoring voxel sizes were $1 \mathrm{~mm}$ in the lung region and $2 \mathrm{~mm}$ in the surrounding water in the $y$ direction (beam's direction). In the $x$ direction, a $2 \mathrm{~mm}$ voxel size was adopted (except for a $1 \mathrm{~mm}$ voxel size used for the $1 \times 1 \mathrm{~cm}^{2}$ field size). In the $z$ direction (the modeled airway/vessel axes' direction), a $2 \mathrm{~mm}$ voxel size was used between -3.1 and $3.1 \mathrm{~cm}$, and a $4 \mathrm{~mm}$ voxel size was used for the remaining lung region. No variance reduction options were used. The $1 \sigma$ statistical uncertainties at the $D_{\max }$ point along the central axis $(\mathrm{CAX})$ are $<0.5 \%$ for all field sizes.

A water phantom of $30 \times 30 \times 20 \mathrm{~cm}^{3}$ with a lung region of $10.2 \times 10.2 \times 10 \mathrm{~cm}^{3}$ embedded was used for simulations (Fig. 1). The front buildup water layer is $5 \mathrm{~cm}$ for the $18 \mathrm{MV}$ beams and $3 \mathrm{~cm}$ for the $6 \mathrm{MV}$ beams. The lung region extends from 5 to $15 \mathrm{~cm}$ for the $18 \mathrm{MV}$ beams and 3 to $13 \mathrm{~cm}$ for the $6 \mathrm{MV}$ beams in the $y$ direction and from -5.1 to $5.1 \mathrm{~cm}$ in both the $x$ and $z$ directions. The surrounding water extends from -15 to $-5.1 \mathrm{~cm}$ and 5.1 to $15 \mathrm{~cm}$ in both the $x$ and $z$ directions.

In the lung region, we first simulated a homogeneous mean density lung and then four heterogeneous lung realizations representing different parts of a real lung. Figures 2(a)-2(f) illustrate how a partial realization was selected from a whole lung realization, as described in Sec. II B. Realization large No. $1\left(L_{1}\right)$ is depicted in Fig. 2(c). This is a magnified view of the lower right framed region in Fig. 2(a). It contains one bronchus, one accompanying artery, and one vein of the same order with diameters larger than $1 \mathrm{~cm}$ (in the range of the orders of "large" bronchi, which include main bronchi and lower lobe bronchus), all close to the central axis region. This is intended as a representative situation in which large structures are all encountered in the middle of the beam's pathway. Realization large No. $2\left[L_{2}\right.$; Fig. 2(d), the upper right framed region in Fig. 2(b)] consists of two large bronchi, arteries, and veins and is intended as a representative situation in which large structures occur off the CAX. Realization small No. $1\left[S_{1}\right.$; Fig. 2(e), the upper left framed region in Fig. 2(a)] and small No. $2\left[S_{2}\right.$; Fig. 2(f), the lower left framed region in Fig. 2(b)] are two variants including only small structures (belonging to the orders of intrasegmental bronchi to terminal bronchioles), which may represent intrasegmental lung regions free of large structures. The results from the four heterogeneous realizations are then compared against the homogenized mean density lung.

We also used a voxel-based MC code, DPM $^{36}$ to perform a preliminary calculation for a voxelized version of one detailed random lung realization, $L_{1}$, for the $6 \mathrm{MV} 1 \times 1$ photon beam. DPM has the same cross section libraries as PENELOPE, and is optimized for medical physics applications. ${ }^{36}$ In our problems, DPM runs about 40 times faster than PENELOPE. For voxelized problems in which the two codes can both be run, they give virtually the same results, and PENELOPE and DPM have both been shown to yield excellent results when compared to experiments. ${ }^{36-39}$ The DPM cutoff energies are the same as PENELOPE, while the DPM step sizes for electrons are $0.5 \mathrm{~cm}$ above $5 \mathrm{MeV}$ and $0.1 \mathrm{~cm}$ otherwise.

\section{RESULTS AND DISCUSSION}

We report the simulation results in the forms of the central axis (CAX) percent depth dose, isodose lines/central dose profiles and the mean lung doses (MLD, which is calculated by dividing the total energy deposited to the lung by the total mass of the lung). All numbers are relative dose normalized to the $D_{\max }$ along the CAX of the mean density case for each field size, respectively. The difference $\Delta(x, y, z)$ between a certain realization and the mean density model is calculated using

$$
\Delta(x, y, z)=\frac{D_{\text {hetero }}(x, y, z)-D_{\text {mean density }}(x, y, z)}{D_{\text {max } \text { mean density }}} .
$$

The absolute values of the $D_{\text {max,mean density, however, are }}$ listed in Table II in units of $\mathrm{MeV} / \mathrm{g} / \mathrm{cm}^{2}$. Due to the existence of a large number of small structures and the fine scoring voxels used in the MC simulations, we have investigated the validity of using a $100 \mathrm{keV}$ electron cutoff energy. The results show no significant differences between a much lower $10 \mathrm{kev}$ and the $100 \mathrm{keV}$ we adopted. Besides the main focus on comparisons between the mean density and the random lungs, we show a comparison between realization $L_{1}$ and its voxelized version in terms of the CAX depth dose.

\section{A. CAX depth dose}

Figures 5 and 6 show the CAX percent depth doses for the 6 and $18 \mathrm{MV}$ photon beams and the $1 \times 1$ and 5 $\times 5 \mathrm{~cm}^{2}$ field sizes, respectively. When the lung is represented by the mean density model, for both energies, the basic shape of the CAX curves is well known: (i) the builddown region upon entering the lung, which is due to a longer secondary electron range in the low-density lung and the loss of charged particle equilibrium (CPE), along with a reduced 
TABLE II. Mean lung dose for each field size $\left(\mathrm{cm}^{2}\right)$ and beam energy for the mean density (MD) lung model and one of the four random heterogeneous realizations as a percentage normalized to the $D_{\max }\left(\mathrm{MeV} / \mathrm{g} / \mathrm{cm}^{2}\right)$ along the central axis of the corresponding mean density lung case. [The mean lung density $\left(\mathrm{g} / \mathrm{cm}^{3}\right)$ for the corresponding case is also listed in the parentheses.]

\begin{tabular}{|c|c|c|c|c|c|c|}
\hline Field size & $\begin{array}{c}D_{\max } \\
\left(\times 10^{-4}\right)\end{array}$ & $\begin{array}{c}\mathrm{MD} \\
(0.26)\end{array}$ & $\begin{array}{c}L_{1} \\
(0.293)\end{array}$ & $\begin{array}{c}L_{2} \\
(0.306)\end{array}$ & $\begin{array}{c}S_{1} \\
(0.242)\end{array}$ & $\begin{array}{c}S_{2} \\
(0.246)\end{array}$ \\
\hline \multicolumn{7}{|c|}{$6 \mathrm{MV}$} \\
\hline $1 \times 1$ & 369 & 0.98 & 1.52 & 0.89 & 0.92 & 0.92 \\
\hline $5 \times 5$ & 16.0 & 22.41 & 24.41 & 21.73 & 21.95 & 22.32 \\
\hline $10 \times 10$ & 4.08 & 77.21 & 77.06 & 76.73 & 77.13 & 77.22 \\
\hline $20 \times 20$ & 1.07 & 83.32 & 82.83 & 82.68 & 83.42 & 83.42 \\
\hline & \multicolumn{6}{|c|}{$18 \mathrm{MV}$} \\
\hline $1 \times 1$ & 571 & 1.29 & 2.01 & 1.19 & 1.22 & 1.22 \\
\hline $5 \times 5$ & 30.2 & 24.12 & 26.47 & 23.68 & 23.50 & 23.92 \\
\hline $10 \times 10$ & 7.67 & 79.69 & 80.17 & 79.82 & 79.32 & 79.52 \\
\hline $20 \times 20$ & 2.01 & 87.84 & 87.60 & 87.31 & 87.81 & 87.87 \\
\hline
\end{tabular}

photon scattering in the low-density medium; and (ii) the buildup region distal to the lung, which is due to the shorter range and the recovery of lateral CPE. These two phenomena become less pronounced and finally disappear as the field size increases because CPE is gradually recovered in the CAX region. The situation for higher energy beams with the same field size is enhanced because the range of the secondary electrons is longer, and thus more volume is needed for compensation. When the mean density lung is replaced by one of the four heterogeneous realizations simulated in this study, deviations of different magnitudes occur, depending on conditions such as whether a large structure is in the beam's path, the size and location of the structure, the material components of the structure, the field size, and the beam's energy.

\section{1. $1 \times 1$ field size}

The most significant perturbations for the $1 \times 1 \mathrm{~cm}^{2}$ field size come from the three large structures in realization $L_{1}$. The dose percent differences in the nonair region are as high as $34 \%$ and $26 \%$ for the 18 and the $6 \mathrm{MV}$ beam, respectively. The buildup and build-down regions within the large structures are clearly visible in Figs. 5(a) and 5(b). The difference is greater in the $18 \mathrm{MV}$ than in the $6 \mathrm{MV}$ beam, due to the longer electron range, causing an enhanced loss of lateral CPE for higher energy photons. The situation is just the opposite with regard to the dose deposited in the airway lumen. In Fig. 5(b) for the $6 \mathrm{MV}$ beam, the airway lumen (air inside) is identified by the lowest dose "valley" (13\% lower than the mean density model), which is less significant in Fig. 5(a) for the $18 \mathrm{MV}$ beam. This is mainly a result of upstream photon scattering, since few secondary electrons originate from within the airway lumen. The same explanation applies to the region between the vein and artery, and the region after the airway, which are mostly occupied by the homogenized background tissue. For realization $L_{2}$ (with large structures mostly outside the beam and only two large veins partly sliced by the beam) and the other two small
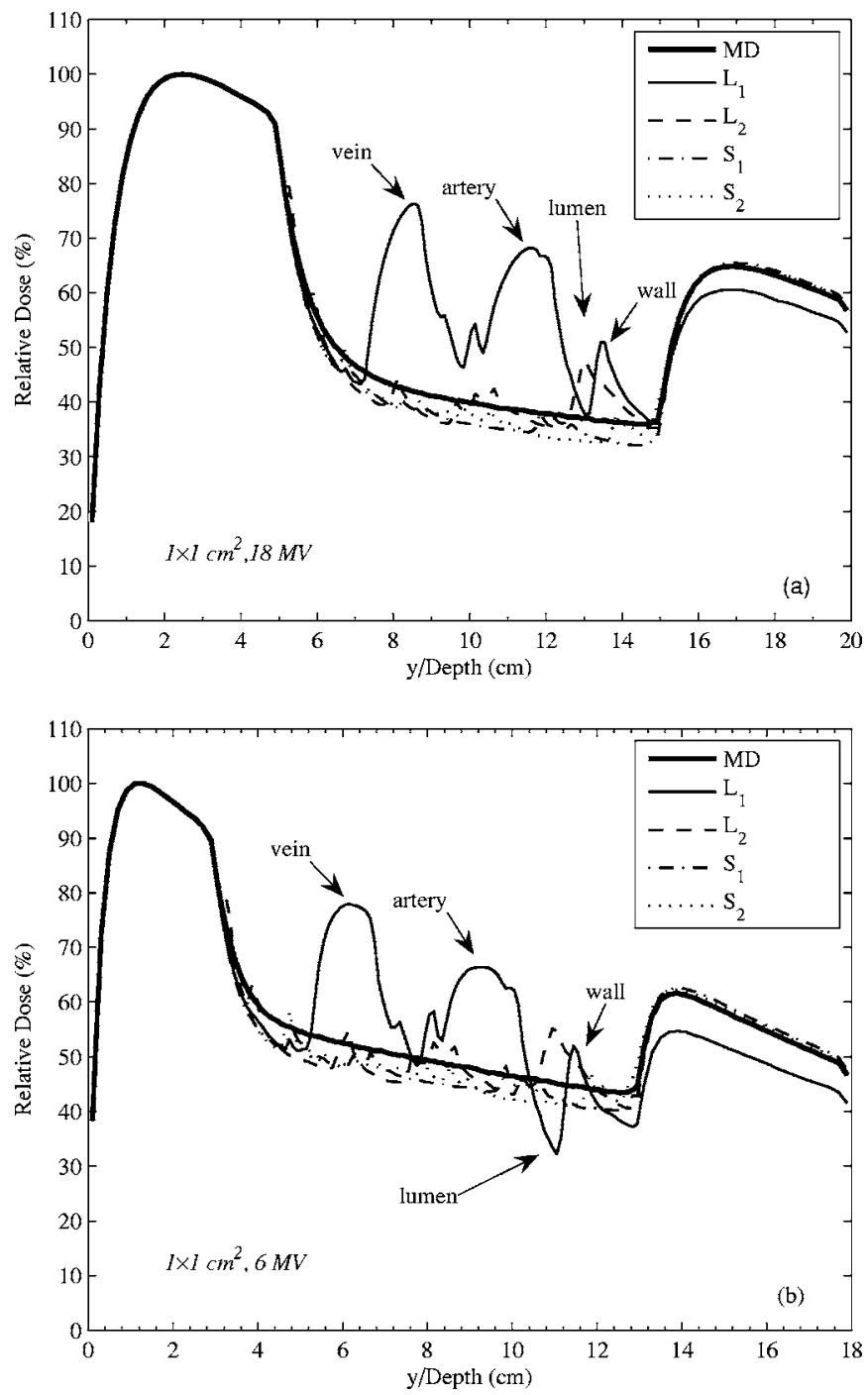

FIG. 5. The CAX percent depth doses for $1 \times 1 \mathrm{~cm}^{2}$ field size and for (a) $18 \mathrm{MV}$, (b) $6 \mathrm{MV}$ beams. The thick solid lines are for the mean density (MD) lung model. The thin solid and the dashed lines are for two large realizations, respectively. The dash-dot and the dotted lines are for two small realizations. All curves for the same field size are normalized to the $D_{\max }$ along the CAX of the corresponding mean density (MD) case. Also indicated in the figures are the large structure locations (an airway, an artery and a vein) on the CAX for realization $L_{1}$.

realizations, smaller perturbations are observed in the beam. For the $1 \times 1$ field size, the difference between the density of the background tissue and that of the mean density lung can cause a significant change in calculated dose. Within the beam, the dose in the small realizations is lower $(<4.5 \%$ and $<5.4 \%$ for the 18 and $6 \mathrm{MV}$ beam) than the mean density case due to the lower density. As a consequence of the extra attenuation of the primary photons caused by large structures (increased local density) inside the beam, and the fact that the dose is dominated by electrons from primary photons, a "shadow" region with reduced dose in the water block distal to the lung appears. This is the case in realization $L_{1}$, where the percent difference is $4.4 \%$ for the $18 \mathrm{MV}$ beam and $7 \%$ for the $6 \mathrm{MV}$ beam. For the other realizations, with most regions inside the beam being low-density background (and 

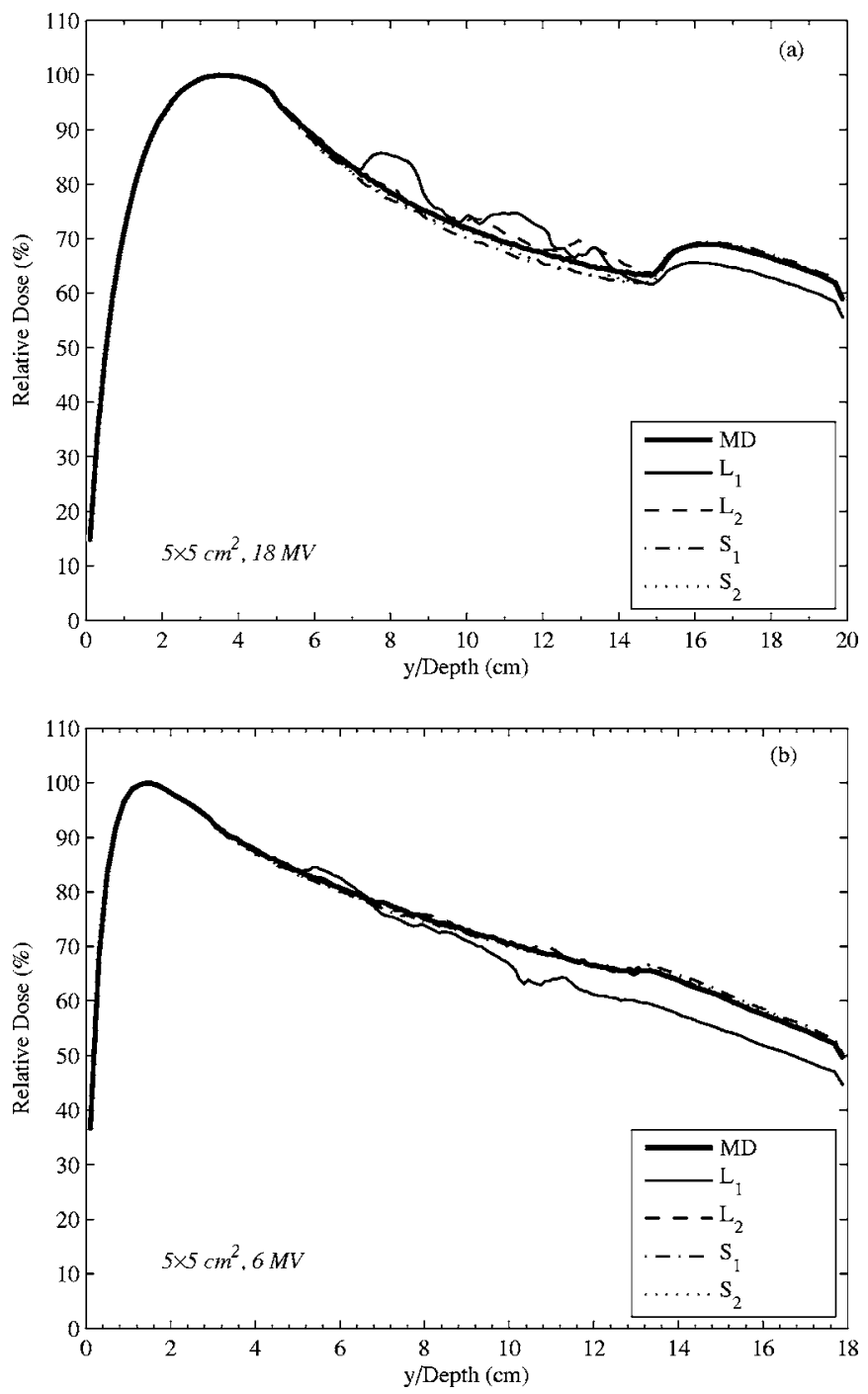

FIG. 6. The CAX percent depth doses for $5 \times 5 \mathrm{~cm}^{2}$ field size and for (a) $18 \mathrm{MV}$, (b) $6 \mathrm{MV}$ beams. The thick solid lines are for the mean density (MD) lung model. The thin solid and the dashed lines are for two large realizations, respectively. The dash-dot and the dotted lines are for two small realizations. All curves for the same field size are normalized to the $D_{\max }$ along the CAX of the corresponding mean density (MD) case.

hence lower dose), the dose in the distal water region is slightly higher $(<1 \%$ for the $18 \mathrm{MV}$ and $<1.5 \%$ for the $6 \mathrm{MV}$ ) than that of the mean density lung. The softer spectrum of the $6 \mathrm{MV}$ beam accounts for the larger differences versus the $18 \mathrm{MV}$ beam.

\section{Larger field sizes}

Figures 6(a) and 6(b) show that with increasing field size, dose perturbations decrease, becoming $7 \%$ and $2 \%$ at the large structures for the $5 \times 5 \mathrm{~cm}$ field size and for the 18 and $6 \mathrm{MV}$ beams, respectively. When the field size exceeds 10 $\times 10 \mathrm{~cm}^{2}$, the differences become $<1.3 \%$ for the 10 $\times 10 \mathrm{~cm}^{2}$, and even smaller for the $20 \times 20 \mathrm{~cm}^{2}$ field size. This is because for the same local density variation inside the beam, the increasing field size leads to gradual recovery of CPE. A similar trend occurs with the shadow region behind the lung with realization $L_{1}$. The percent differences in the region distal to the lung for the 18 and $6 \mathrm{MV}$ are $<3.9 \%$ and $<6.2 \%$ for the $5 \times 5,<3.8 \%$ and $<6 \%$ for the $10 \times 10$ and $<3.8 \%$ and $<5.9 \%$ for the $20 \times 20 \mathrm{~cm}^{2}$ beam, respectively. However, these changes as a function of field size are less than those within the large structures. This indicates that even though CPE exists in the CAX and local perturbations are negligible, the accumulated attenuation by the upstream structures is still present. The magnitude of the differences is not sensitive to the field size but is mainly determined by the structures in the beam's pathway. For the cases in which the tumor is on the distal side of a large structure in the beam's pathway, simply increasing the field size may not be an effective way to increase the dose to the tumor. For the two small realizations without significant large local density variations, the differences in the lung from the mean density model are small, even for the $5 \times 5 \mathrm{~cm}^{2}$ field size $(<2.3 \%$ for the $18 \mathrm{MV}$ beam and $<1 \%$ for the $6 \mathrm{MV}$ ).

\section{B. Isodose lines and profiles}

\section{1. $1 \times 1$ field size}

Figures 7(a) and 7(b) show the isodose lines for the mean density model and realization $L_{1}$ on the $x-y$ plane at $z=0$ for the $1 \times 1 \mathrm{~cm}^{2}$ field size and for the $6 \mathrm{MV}$ energy. Two central dose profiles at selected depths are also shown in Figs. $8(\mathrm{a})$ and $8(\mathrm{~b})$. The selected depths are (i) $3.1 \mathrm{~cm}$ deep in the lung (i.e., $y=6.1 \mathrm{~cm}$ ), crossing the large vein; (ii) $7.9 \mathrm{~cm}$ deep in the lung (i.e., $y=10.9 \mathrm{~cm}$ ), crossing the airway lumen. The purpose of presenting isodose lines and central dose profiles together is to provide a more complete picture of the perturbations to the dose distribution caused by the structures in the lung, while simultaneously giving typical depth information.

The deviation from the mean density model is that the smoothness of the isodose lines is altered, due to local density variations from randomly positioned structures. Apart from the mean density model, large solid structures inside the beam attenuate more primary photons and become additional local secondary particle "sources;" while at regions free of these structures, lower dose occurs due to the lower density of the background. These result in either the broadening or contraction of the penumbra region, as is clearly indicated by the $10 \%$ and 5\% isodose lines in Fig. 7(b). A similar result is also recognized with the $18 \mathrm{MV}$ beam. Also, a structure can increase or decrease the local dose, depending on whether it is tissue or air, with the extent of distortion depending on the size and location of the structure. Compared to the mean density model, two hot spots are present in Fig. 7(b) for realization $L_{1}$ (similar hot spots appear for realization $L_{2}$, as well). This is also the case for the $18 \mathrm{MV}$ beam. These can also be seen in Fig. 8(a), which shows at $3.1 \mathrm{~cm}$ deep in the lung, the large vein is almost centered on the CAX; therefore a nearly symmetric profile for realization $L_{1}$ occurs. In the profile for realization $L_{2}$ in Fig. 8(a), the peak corresponds to a small vein with diameter about $0.22 \mathrm{~cm}$, located at about $x=-0.125 \mathrm{~cm}$ and totally inside the beam. For the small $1 \times 1$ field size, no CPE exists inside the 

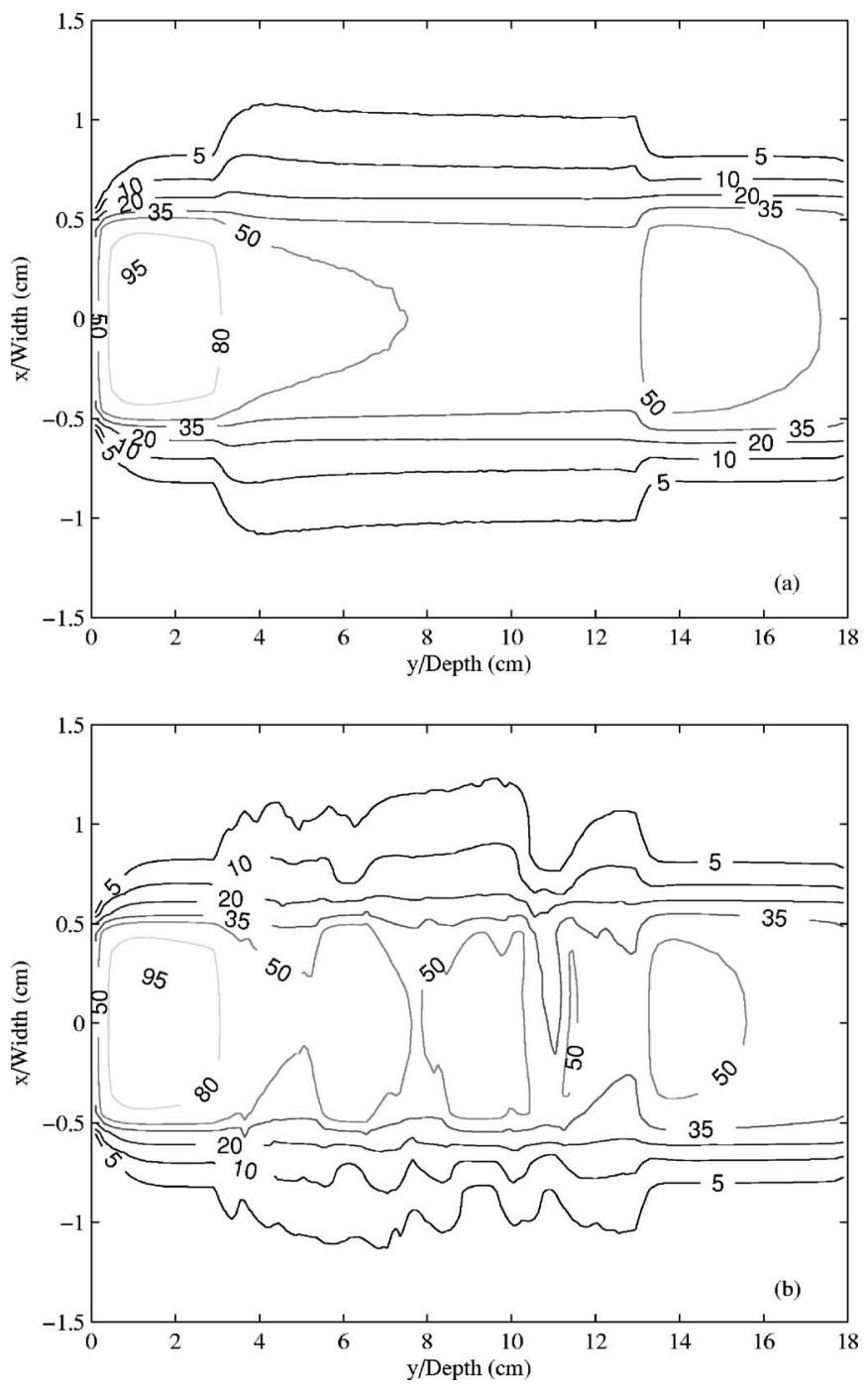

FIG. 7. Isodose lines on $x-y$ plane $(z=0)$ for $1 \times 1 \mathrm{~cm}^{2}$ field size for (a) mean density model, $6 \mathrm{MV}$; (b) realization $L_{1}, 6 \mathrm{MV}$. The abscissa is in $y$ direction and the ordinate is in $x$ direction.

beam, so even a small structure perturbs the dose significantly. The same situation applies to the profiles at depth $7.9 \mathrm{~cm}$ deep in the lung [Fig. 8(b)], where the large airway's wall and lumen in realization $L_{1}$ are indicated with the clear asymmetry.

\section{Larger field sizes}

As discussed in Sec. IV A 2, CPE is gradually recovered inside the beam with increased field sizes. For the 10 $\times 10 \mathrm{~cm}^{2}$ field size and for both energies, the differences between the mean density model and all four realizations are negligible $(<1.5 \%$ on average) at the high dose region, except for the middle (realization $L_{1}$ ), due to the extra upstream attenuation. The large structures off the CAX yet inside the $10 \times 10 \mathrm{~cm}^{2}$ field size in realization $L_{2}$ lead to a similar but smaller attenuation effect, which is clearer for the softer $6 \mathrm{MV}$ beam ( $\sim 4 \%$ at $1.1 \mathrm{~cm}$ behind the lung) than the $18 \mathrm{MV}$ beam $(\sim 2.5 \%$ at the same depth). Although the lateral CPE is well established deep inside such a wide beam,
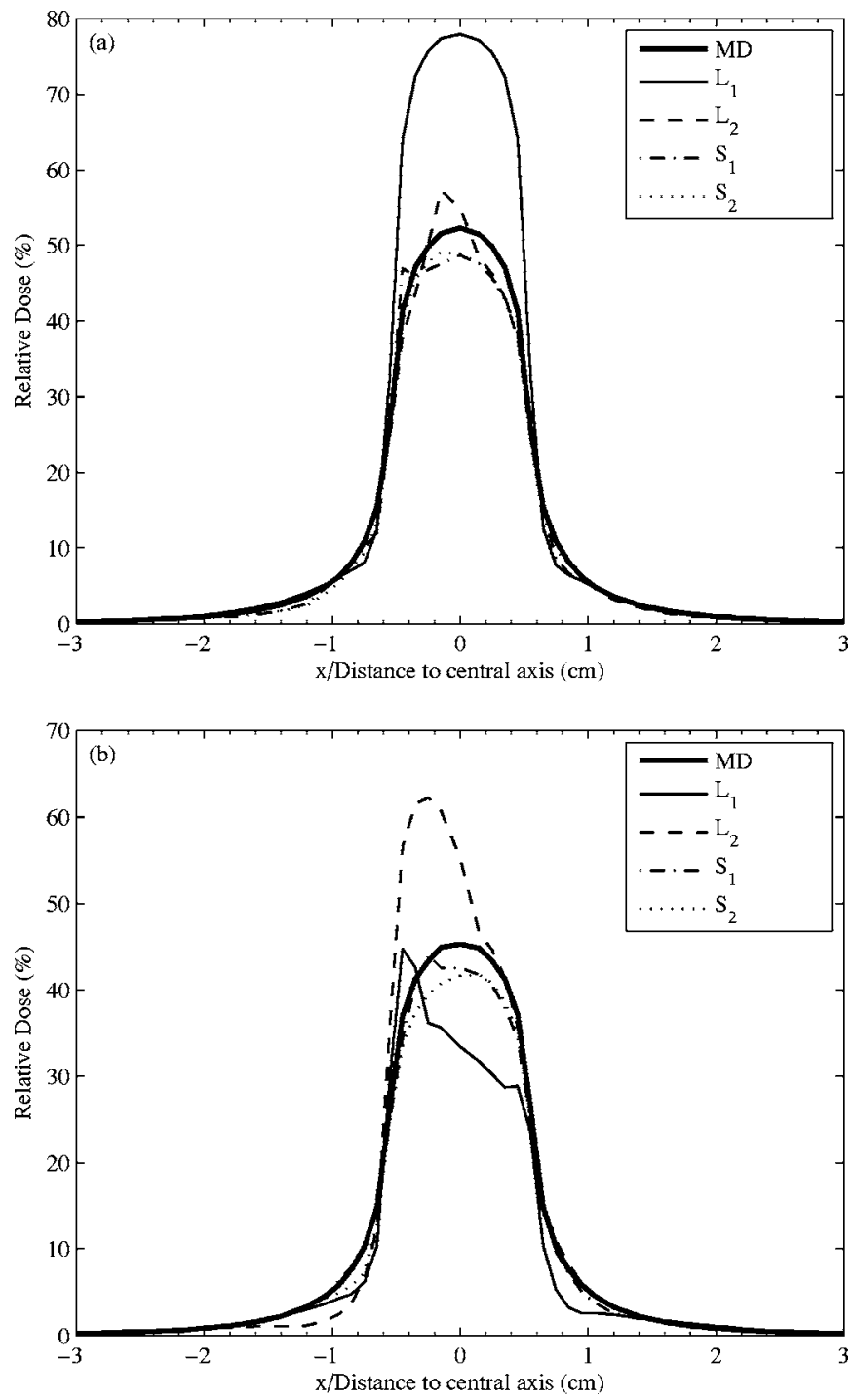

FIG. 8. CAX dose profiles for $1 \times 1 \mathrm{~cm}^{2}$ field size for $6 \mathrm{MV}$ at (a) $y$ $=6.1 \mathrm{~cm}$; (b) $y=10.9 \mathrm{~cm}$. The thick solid lines are for the mean density (MD) lung model. The thin solid and the dashed lines are for two large realizations, respectively. The dash-dot and the dotted lines are for two small realizations. All curves for the same field size are normalized to the $D_{\max }$ along the CAX of the corresponding mean density (MD) case.

in regions close to the beam's edge, lateral CPE does not exist because there is no compensation scattering from outside. Therefore, any significant local density variations occurring close to the beam's edge may possibly be of concern. To investigate this, we examined the central dose profiles in realization $L_{2}$ for the $10 \times 10 \mathrm{~cm}^{2}$ field size at two different depths (6.1 and $7.5 \mathrm{~cm}$ deep in the lung), in which the first depth crosses the two large airways' lumens and the second depth crosses the two accompanying arteries and a single vein near the CAX. The two airways and their arteries are close to the lung-tissue interface $(<1 \mathrm{~cm})$. However, only slight local perturbations $(<2 \%)$ are found to be associated with these structures, which indicates a state close to CPE. For this large field size, the increased scattering within water may be compensating for the dose reduction in the large structures. 


\section{Mean lung doses}

The mean lung dose (MLD) can illustrate from another point of view the perturbations caused by the random structures. Table II gives the MLDs for each geometry and field size, for both the 18 and $6 \mathrm{MV}$ energies. We observe: (i) the MLDs are not directly related to the mean density of the whole lung. Rather, they are mainly determined by the tissue of the region through which the beam passes. For example, the $L_{1}$ and $L_{2}$ realizations have almost the same whole lung mean density (with a mean lung density of 0.293 and $0.306 \mathrm{~g} / \mathrm{cm}^{3}$, respectively, both greater than the mean density model's $0.26 \mathrm{~g} / \mathrm{cm}^{3}$ ) but differ much in structures in the narrow central regions covered by the $1 \times 1 \mathrm{~cm}$ field. Along the CAX, realization $L_{1}$ has three large structures, but realization $L_{2}$ has mostly small background structures. For the $6 \mathrm{MV}$ beam, this difference results in a much higher $(55 \%$ larger than the mean density model) MLD for the $L_{1}$ realization and a significantly lower (9.2\% smaller) MLD for the $L_{2}$ realization. This can also be seen in more detail from the corresponding CAX depth doses and the central dose profiles, which show that most energy is deposited within the beam and in the high density regions (for the $1 \times 1 \mathrm{~cm}^{2}$ field size). (ii) As the field size increases, the differences between the MLDs for the two large realizations decrease for 5 $\times 5 \mathrm{~cm}^{2}$ field size and become negligible for the $10 \times 10$ and $20 \times 20 \mathrm{~cm}^{2}$ field sizes. The differences between the four heterogeneous realizations and the mean density model also show a similar trend. Two reasons contribute to this (as a function of field size): (a) more structures are present in the open beam, so more energy is absorbed; and (b) the gradual recovery of CPE inside the beam, (iii) At all field sizes and beam energies, the MLDs of the two small realizations (with a mean lung density of 0.242 and $0.246 \mathrm{~g} / \mathrm{cm}^{3}$, respectively, both $<$ the mean density model's $0.26 \mathrm{~g} / \mathrm{cm}^{3}$ ) are much closer to each other and also closer to that of the mean density model than those of the two large realizations.

\section{Detailed vs voxelized lungs}

Figure 9 is a preliminary calculation showing the difference between a detailed random lung realization $L_{1}$ [Fig. 2(c)] and its voxelized version (Fig. 4) in terms of CAX depth dose. Basically, the two curves agree reasonably well with each other. The voxelized lung at the resolution of 0.4 $\times 0.4 \times 0.4 \mathrm{~cm}^{3}$ reveals most of the structures in the detailed one, and in particular, the magnitude of the underdosing distal to the lung is reproduced. This is as expected, because the underdosing is almost entirely affected by the amount of attenuation of the primary photons, which in turn depends on the radiological length along the beam's path. The average density in the beam's pathway is conserved, even though the homogenization in each voxel tends to smooth out the details of the structure. However, the voxelization still causes local differences up to $5 \%$ in the nonair region (up to $12 \%$ in the airway), which is due to the smoothing of the structures in the voxels. Appropriate resolutions of voxelization is a complex issue and subject to further investigation. We will report more comprehensive results in the near future.

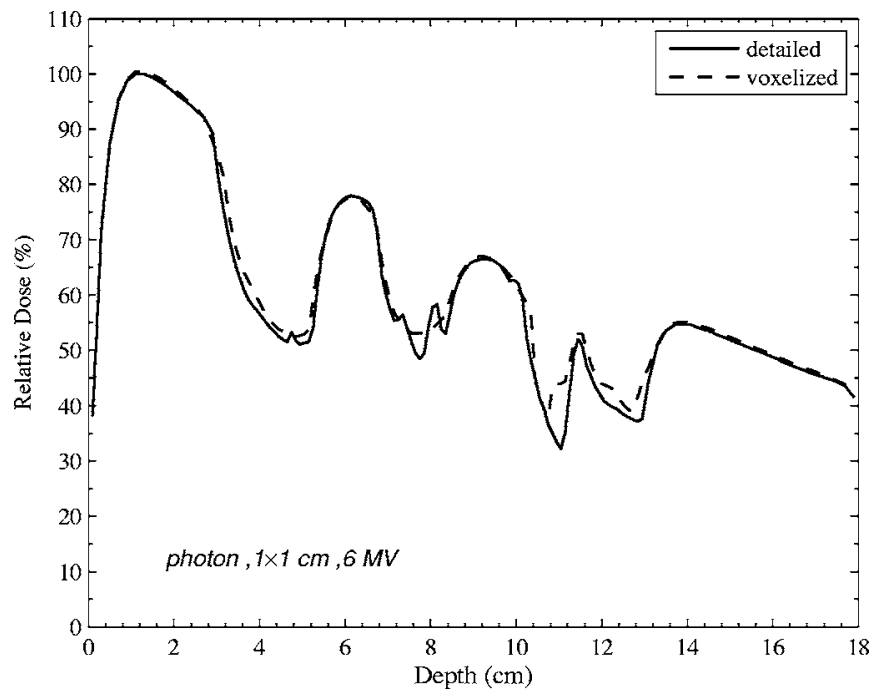

FIG. 9. The CAX percent depth doses for a $1 \times 1 \mathrm{~cm}^{2}$ field size and $6 \mathrm{MV}$ beam. The solid line is for the detailed realization $L_{1}$ [Fig. 2(c)] and the dash line is for its voxelized version (Fig. 4), respectively. Both curves are normalized to the $D_{\max }$ along the CAX of the detailed $L_{1}$ case.

\section{CONCLUSION}

We have developed a random heterogeneous 2 1/2D lung model, based upon real lung physical data, by explicitly treating the bronchial and vessel tree structures within a homogenized tissue background with adjusted density. Four realizations of this model were chosen to represent various scenarios that may be encountered in lung treatment planning. Monte Carlo simulations using the PENELOPE Monte Carlo code were performed on the homogeneous mean density lung model and the four heterogeneous realizations, for a single beam of two different energies (6 and $18 \mathrm{MV})$ and four field sizes $\left(1 \times 1,5 \times 5,10 \times 10\right.$ and $\left.20 \times 20 \mathrm{~cm}^{2}\right)$. By comparing the CAX percent depth doses, the central dose profiles, and the MLD among all the cases, we conclude that when the beam traverses a region with significantly large local structures, such as the regions close to the main and lobar bronchi and the vessels of the same order, a serious concern can exist if these structures are inside the beam. Also, significant local perturbations in dose (more than $30 \%$ of $D_{\max }$ larger than the mean density model for the $18 \mathrm{MV}$ ) were found for the small $1 \times 1 \mathrm{~cm}^{2}$ field size. As the field size increases, the local perturbation may finally vanish as CPE is established. However, the extra concentration of density inside the beam can lead to dose reduction as high as 7\% of $D_{\max }$ in the distal shadow part of the beam, which is not compensated by inward scattering, even with the largest field size $\left(20 \times 20 \mathrm{~cm}^{2}\right)$ in this study. This situation affects lowenergy beams more than high-energy ones because of their softer spectra. Also, the reduction in dose in the shadow regions behind large structures is largely independent of the field size. On the other hand, if there are no large structures inside the beam, the results (especially the MLD) are closer to the mean density model (yet still show geometry-specific variation). For the small field sizes, such as the $1 \times 1 \mathrm{~cm}^{2}$ beam where lateral $\mathrm{CPE}$ is absent, even a relatively small 
structure $(\sim 0.22 \mathrm{~cm})$ simulated in the heterogeneous model can significantly perturb the dose.

Also, in a preliminary calculation, we compared the CAX depth dose for a random lung and a voxelized counterpart using a $0.4 \mathrm{~cm}$ resolution. We found up to a $5 \%$ of $D_{\max }$ difference in nonair region.

Our results show that the mean density model for the whole lung is not generally a good approximation, especially for small field sizes, and that a voxelized model with $0.4 \mathrm{~cm}$ resolution can also have significant errors.

In future work, we plan to extend our random lung simulations to more realistic geometries, in which the lung, tumor, and surrounding tissue are more accurately represented, and in which setup errors and patient breathing are included. We also plan to systematically study the accuracy of dose calculations obtained by Monte Carlo on voxelized versions of the random lung at different resolutions. (In other words, we plan to assess the errors in dose due to voxelization.) The effect of voxelization on dose volume histograms and calculation times has been studied recently. ${ }^{40}$ Also, comparisons have recently been made between dose calculations obtained by Monte Carlo, convolution/superposition, and pencil beam methods for voxelized lung intensity modulated radiation therapy. ${ }^{41}$ By examining similar issues in our work, it should ultimately be possible to assess the accuracy of current dose calculation methods (i.e., convolution/superposition and pencil beam) for the lung. The work presented in this paper is a first step toward achieving this practical objective.

\section{ACKNOWLEDGMENTS}

Part of this work was performed under U.S. Government Contract No. DE-AC52-06NA25396 for Los Alamos National Laboratory, which is operated by the Los Alamos National Security (LANS) LLC for the U.S. Department of Energy.

\footnotetext{
a) Author to whom correspondence should be addressed. Electronic mail: lliang@umich.edu

${ }^{1}$ C. G. Orton, P. M. Mondalek, J. T. Spicka, D. S. Herron, and L. I. Andres, "Lung corrections in photon beam treatment planning: Are we ready?" Int. J. Radiat. Oncol., Biol., Phys. 10, 2191-2199 (1984).

${ }^{2}$ A. van't Riet, H. C. Stam, A. C. Mak, and F. H. van Slooten, "Implications of lung corrections for dose specification in radiotherapy," Int. J. Radiat. Oncol., Biol., Phys. 11, 621-625 (1985).

${ }^{3}$ W. G. McKenna, K. Yeakel, A. Klink, B. A. Fraass, J. van de Geijn, E. Glatstein, and A. S. Lichter, "Is correction for lung density in radiotherapy treatment planning necessary?" Int. J. Radiat. Oncol., Biol., Phys. 13, 273-278 (1987).

${ }^{4} \mathrm{~K}$. Mah and J. Van Dyk, "On the impact of tissue inhomogeneity corrections in clinical thoracic radiation therapy," Int. J. Radiat. Oncol., Biol., Phys. 21, 1257-1267 (1991).

${ }^{5}$ Photon Treatment Planning Collaborative Working Group, "Role of inhomogeneity corrections in three-dimensional photon treatment planning," Int. J. Radiat. Oncol., Biol., Phys. 21, 59-69 (1991).

${ }^{6}$ C. G. Orton, S. Chungbin, E. E. Klein, M. T. Gillin, T. E. Schultheiss, and W. T. Sause, "Study of lung density corrections in a clinical trial (rtog 88-08) radiation therapy oncology group," Int. J. Radiat. Oncol., Biol., Phys. 4, 787-794 (1998).

${ }^{7}$ S. J. Frank, K. M. Forster, C. W. Stevens, J. D. Cox, R. Komaki, Z. Liao, S. Tucker, X. Wang, R. E. Steadham, C. Brooks, and G. Starkschall, "Treatment planning for lung cancer: Traditional homogeneous pointdose prescription compared with heterogeneity-corrected dose-volume prescription,” Int. J. Radiat. Oncol., Biol., Phys. 56, 1308-1318 (2003).
}

${ }^{8}$ T. R. Mackie, E. el Khatib, J. Battista, J. Scrimger, J. Van Dyk, and J. R. Cunningham, "Lung dose corrections for 6 and $15 \mathrm{MV}$ x rays," Med. Phys. 12, 327-332 (1985).

${ }^{9}$ R. K. Rice, B. J. Mijnheer, and L. M. Chin, "Benchmark measurements for lung dose corrections for X-ray beams," Int. J. Radiat. Oncol., Biol., Phys. 15, 399-409 (1988).

${ }^{10}$ T. Knöös, A. Ahnesjo, P. Nilsson, and L. Weber, "Limitations of a pencil beam approach to photon dose calculations in lung tissue," Phys. Med. Biol. 40, 1411-1420 (1995).

${ }^{11}$ P. J. White, R. D. Zwicker, and D. T. Huang, "Comparison of dose homogeneity effects due to electron equilibrium loss in lung for 6 and 18 MV photons," Int. J. Radiat. Oncol., Biol., Phys. 34, 1141-1146 (1996).

${ }^{12}$ R. C. Miller, J. A. Bonner, and R. W. Kline, "Impact of beam energy and field margin on penumbra at lung tumor-lung parenchyma interfaces," Int. J. Radiat. Oncol., Biol., Phys. 41, 707-713 (1998).

${ }^{13}$ M. J. Brugmans, A. van der Horst, J. V. Lebesque, and B. J. Mijnheer, "Beam intensity modulation to reduce the field sizes for conformal irradiation of lung tumors: A dosimetric study," Int. J. Radiat. Oncol., Biol., Phys. 43, 893-904 (1999).

${ }^{14}$ M. Engelsman, E. M. Damen, P. W. Koken, A. A. van't Veld, K. M. van Ingen, and B. J. Mijnheer, "Impact of simple tissue inhomogeneity correction algorithms on conformal radiotherapy of lung tumours," Radiother. Oncol. 60, 299-309 (2001).

${ }^{15}$ P. Carrasco, N. Jornet, M. A. Duch, L. Weber, M. Ginjaume, T. Eudaldo, D. Jurado, A. Ruiz, and M. Ribas, "Comparison of dose calculation algorithms in phantoms with lung equivalent heterogeneities under conditions of lateral electronic disequilibrium," Med. Phys. 31, 2899-2911 (2004). ${ }^{16}$ I. J. Chetty, M. Rosu, D. L. McShan, B. A. Fraass, and R. K. Ten Haken, "The influence of beam model differences in the comparison of dose calculation algorithms for lung cancer treatment planning," Phys. Med. Biol. 50, 801-815 (2005).

${ }^{17}$ G. C. Pomraning, Linear Kinetic Theory and Particle Transport in Stochastic Mixtures, p. 106 (World Scientific, Singapore, 1991).

${ }^{18}$ J. E. Cotes and G. L. Leathart, Lung function: Assessment and Application in Medicine, 5th ed. (Blackwell, Oxford, 1993).

${ }^{19}$ J. A. McDonald, Lung Growth and Development (Dekker, New York, 1997).

${ }^{20}$ L. Dumas and F. Golse, "Homogenization of transport equations," SIAM J. Appl. Math. 60, 1447-1470 (2000).

${ }^{21}$ E. R. Weibel, Morphometry of the Human Lung (Springer, Berlin, 1963).

${ }^{22} \mathrm{~K}$. Horsfield and G. Cumming, "Morphology of the bronchial tree in man," J. Appl. Physiol. 24, 373-383 (1968).

${ }^{23}$ K. Horsfield, G. Dart, D. E. Olson, G. F. Filley, and G. Cumming, "Models of the human bronchial tree," J. Appl. Physiol. 31, 207-217 (1971).

${ }^{24}$ H. Parker, K. Horsfield, and G. Cumming, "Morphology of distal airways in the human lung," J. Appl. Physiol. 31, 386-391 (1971).

${ }^{25}$ B. Haefeli-Bleuer and E. R. Weibel, "Morphometry of the human pulmonary acinus," Anat. Rec. 220, 401-414 (1988).

${ }^{26}$ W. Huang, R. T. Yen, M. McLaurine, and G. Bledsoe, "Morphometry of the human pulmonary vasculature," J. Appl. Physiol. 81, 2123-2133 (1996).

${ }^{27}$ M. H. Xawhai, P. Hunter, J. Tschirren, J. Reinhardt, G. McLennan, and E. A. Hoffman, "Ct-based geometry analysis and finite element models of the human and ovine bronchial tree," J. Appl. Physiol. 97, 2310-2321 (2004).

${ }^{28}$ S. Matsuoka, K. Uchiyama, H. Shima, N. Ueno, S. Oish, and Y. Nojiri, "Bronchoarterial ratio and bronchial wall thickness on high-resolution ct in asymptomatic subjects: Correlation with age and smoking," AJR, Am. J. Roentgenol. 180, 513-518 (2003).

${ }^{29}$ E. W. Larsen and L. Liang, "The atomic mix approximation for charged particle transport," SIAM J. Appl. Math. (to appear).

${ }^{30} \mathrm{D}$. Liljequist and M. Ismail, "Transport mean free path related to trajectory patterns: Comparison of nonrelativistic and highly relativistic electron penetration through matter," J. Appl. Phys. 62, 342-350 (1987).

${ }^{31}$ D. Liljequist, M. Ismail, F. Salvat, R. Mayol, and J. D. Martinez, "Transport mean free path tabulated for the multiple elastic scattering of electrons and positrons at energies $\leq 20 \mathrm{MeV}$," J. Appl. Phys. 68, 3061-3065 (1990).

${ }^{32}$ L. Liang, Development and Application of a Random Lung Model for Dose Calculations in Radiotherapy, Ph.d. Thesis, University of Michigan (2007).

${ }^{33}$ J. Van Dyk, T. J. Keane, and W. D. Rider, "Lung density as measured by 
computerized tomography: Implications for radiotherapy,” Int. J. Radiat. Oncol., Biol., Phys. 8, 1363-1372 (1982).

${ }^{34}$ F. Salvat, J. M. Fernandez-Varea, and J. Sempau, PENELOPE, a code system for Monte Carlo simulation of electron and photon transport (OECD Nuclear Energy Agency, 2003).

${ }^{35}$ D Sheikh-Bagheri and D. W. Rogers, "Monte Carlo calculation of nine megavoltage photon beam spectra using the beam code," Med. Phys. 29, 391-402 (2002).

${ }^{36}$ J. Sempau, S. J. Wilderman, and A. F. Bielajew, "DPM, a fast, accurate Monte Carlo code optimized for photon and electron radiotherapy treatment planning dose calculations," Phys. Med. Biol. 45, 2263-2291 (2000).

${ }^{37}$ I. J. Chetty, J. M. Moran, D. L. McShan, B. A. Fraass, S. J. Wilderman, and A. F. Bielajew, "Benchmarking of the dose planning method (DPM) Monte Carlo code using electron beams from a racetrack microtron," Med. Phys. 29, 1035-1041 (2002).
${ }^{38}$ I. J. Chetty, J. M. Moran, T. S. Nurushev, D. L. McShan, B. A. Fraass, S. J. Wilderman, and A. F. Bielajew, "Experimental validation of the DPM Monte Carlo code using minimally scattered electron beams in heterogeneous media," Phys. Med. Biol. 47, 1837-1851 (2002).

${ }^{39}$ I. J. Chetty, P. M. Charland, N. Tyagi, D. L. McShan, B. A. Fraass, and A. F. Bielajew, "Photon beam relative dose validation of the DPM Monte Carlo code in lung-equivalent media," Med. Phys. 30, 563-573 (2003).

${ }^{40}$ B. De Smedt, B. Vanderstraeten, N. Reynaert, W. De Neve, and H. Thierens, "Investigation of geometrical and scoring grid resolution for Monte Carlo dose calculations for IMRT," Phys. Med. Biol. 50, 4005-4019 (2005).

${ }^{41}$ B. Vanderstraeten, N. Reynaert, L. Paelinck, I. Madani, C. De Wagter, W. De Gersem, W. De Neve, and H. Thierens, "Accuracy of patient dose calculation for lung IMRT: A comparison of Monte Carlo, convolution/ superposition, and pencil beam computations," Med. Phys. 33, 31493158 (2006). 